\title{
Encephalopathy and severe neuropathy due to probable systemic vasculitis as an initial manifestation of mixed connective tissue disease
}

\author{
Hideaki Matsui, Fukashi Udaka, Masaya Oda, Tamotsu Kubori, Kazuto Nishinaka, \\ Masakuni Kameyama \\ Department of Neurology, Sumitomo Hospital, Osaka, Japan
}

\begin{abstract}
We described a 69-year-old woman with neurological manifestations due to mixed connective tissue disease (MCTD). The patient demonstrated subacute cognitive decline, seizure and gait disturbance with no connective tissue manifestation. She had been diagnosed with dementia at another hospital, later in our hospital, serological examinations disclosed high titers of anti-RNP antibody. Cognitive dysfunction in this patient was dramatically ameliorated by steroid therapy. Three months later, she developed edema of the hands, synovitis and acrosclerosis. The patient was finally diagnosed as having MCTD. We emphasized MCTD as a rare cause of "treatable dementia".
\end{abstract}

Key words: Mixed connective tissue disease, treatable dementia, cognitive dysfunction

The occurrence of neurological or psychiatric disturbances in association with rheumatic diseases has been an area of considerable interest for many years. ${ }^{[1]}$ Mixed connective tissue disease (MCTD) is characterized by clinical features of systemic lupus erythematosus (SLE), scleroderma and polymyositis occurring simultaneously or sequentially, in the presence of high titers of antibody to an Rnase-sensitive ribonuclear protein complex, U1 small nuclear RNP (ribonucleoprotein). Neurological problems in MCTD are seen in approximately 10 20\% of cases; ${ }^{[2]}$ however, it is difficult to diagnose patients with just neurological manifestations. We present a patient demonstrating subacute cognitive dysfunction, seizure and gait disturbance with no connective tissue manifestation. The patient was later disclosed as having MCTD. Cognitive dysfunction and seizure in this patient were ameliorated by steroid therapy. We emphasize MCTD as a rare cause of "treatable dementia".

\section{Case Report}

A 69-year-old woman demonstrated gait disturbance from April 2004 and the gait disturbance gradually and symmetrically progressed. The patient had no congenital disorders, no regular medicine, no history of diseases such as hypertension, diabetes mellitus or sleep disorders, no history of toxin exposure and no smoking or alcohol. In October 2004, cognitive dysfunction appeared and subacutely deteriorated. She also demonstrated aphasia and experienced transient seizure once in the previous month. The patient had been diagnosed as having dementia in another hospital, but in November 2004 was admitted to our hospital. On admission, the patient was alert and awake. She could not stay standing nor walk. There were no trophic changes in the feet. She had cognitive impairment, mainly memory disturbance. The score on a Mini-mental state examination (MIMSE) was 10/30. She demonstrated incomplete transcortical motor aphasia, and sometimes palilalia, but apraxia or agnosia was not seen. Cranial nerves were intact except for right facial sensory disturbance in touch, pain and temperature. Muscle strength in upper limbs was slightly diminished, while in lower limbs all muscles revealed distal dominant weakness, as reflected by the 3 score on a manual muscle test. There was moderate atrophy in bilateral tibialis anterior muscles. Deep tendon reflexes were all symmetrical and normal in upper limbs but were absent in the lower limbs. Bilateral palmomental reflexes were positive, as was right Babinski's reflex, while left plantar reflex was equivocal. Vibration, touch, pain and temperature senses in the bilateral lower limbs were greatly and symmetrically diminished. Voluntary pain was not seen. There was no sensory level in the distribution 
of sensory disturbances. The neuropathy was painless. No cerebellar or autonomic manifestations were present. Neck stiffness and Kernig's sign were negative. The patient did not show any symptoms in connective tissues such as Raynaud's syndrome, synovitis, acrosclerosis, myositis, edema or sclerodactylia

Blood analysis disclosed mild inflammatory responses, and high indexes of SS (Sjögren syndrome) -A, RNP and TPO (thyroid peroxidase $=$ thyroid myeloperoxidase) antibody, and hypothyroidism. Other antibodies such as DNA, SS-B, cardiolipin, Sm, or ANCA (antineutrophil cytoplasmic antibodies) were not detected. The tests for lupus anticoagulant were not performed. Antinuclear antibodies were detected at low titers. Vitamin B1, B12 and folic acid were normal. Hepatitis B surface antigen, Hepatitis $\mathrm{C}$ antibody, rapid plasma reagin test and treponemal tests: hemagglutination test were all negative. Cerebrospinal fluid examinations showed high total protein and increased cell counts. No bacteria or malignant cells were detected, neither was tubercle bacillus on a culture test and by DNA-PCR. Oligoclonal bands were positive while myelin basic protein was not detected. Herpes simplex virus, herpes zoster virus, mumps, measles and toxoplasma were negative during the entire clinical course. Urine examinations were normal. Ultrasonic cardiography demonstrated pulmonary hypertension (Tricuspid regurgitation peak pressure gradient $=37 \mathrm{mmHg}$ ). Chest computed tomography (CT) was normal. Magnetic resonance imaging (MRI) showed multiple high intensity signals in T2 and FLAIR sequences [Figure 1]. These lesions were not enhanced. Brain and cervical magnetic resonance angiography did not show any abnormal lesions. Electroencephalography demonstrated diffuse slow activity. Motor action potentials in the bilateral tibial nerves were not evoked, while a motor conduction study demonstrated low amplitude (amplitude: $0.6 \mathrm{mV}$, conduction velocity: $30 \mathrm{~ms}$ ) in the bilateral peroneal nerves. Motor action potentials in the bilateral ulnar nerves were intact and those in the bilateral median nerves showed slightly delayed distal latency. Action potentials in the bilateral sural nerves were not evoked, and somatosensory evoked potentials could not be detected. There was no evidence of conduction block. These nerve conduction studies demonstrated the mononeuritis multiplex pattern; severe damage in the tibial nerves in contrast to relative preservation of the peroneal nerves, which are more prone to be impaired by polyneuropathic pathology. Blink reflex test disclosed right trigeminal neuropathy [Figure 2]. No malignant tumor was detected on chest and abdominal CT, endoscopy, sonography and tumor marker studies.

The patient was tentatively diagnosed as having neurological deteriorations due to MCTD. Corticosteroid pulse therapy was given and thereafter $60 \mathrm{mg} /$ day prednisolone was prescribed orally. Oral prednisolone was gradually tapered and her cognitive function improved. MMSE score was $24 / 30$ one month after therapy. Aphasia had completely disappeared, and there were no seizures after treatment. However the trigeminal and peripheral neuropathies, and gait disturbance did not ameliorate, and brain MRI did not change significantly. Inflammatory response in blood and cerebrospinal fluid disappeared. Three months after admission, she gradually developed edema of the hands, synovitis and acrosclerosis. The patient was finally diagnosed as having. MCTD. The patient did not show remarkable clinical change in further three months after the final diagnosis.

\section{Discussion}

The patient fulfilled the diagnostic criteria of MCTD by Japanese study group ${ }^{[2]}$ and probable diagnosis by Sharp. ${ }^{[3]}$ While we could not exclude Hashimoto's encephalopathy, MCTD was strongly suggested by the concomitant pulmonary hypertension, trigeminal neuropathy and peripheral neuropathy. Especially, trigeminal neuropathy has been reported to have a close relation with MCTD compared to other collagen disease or Hashimoto's disease, ${ }^{[4,5]}$ therefore this suggested strongly that neurological manifestations in this patients were due to MCTD. Pulmonary hypertension also has been considered as a unique complication of MCTD,${ }^{[6]}$ however some authors pointed out the correlation between thyroid dysfunction and pulmonary hypertension. ${ }^{[7]}$ It is unnatural to consider encephalopathy was due to Hashimoto's

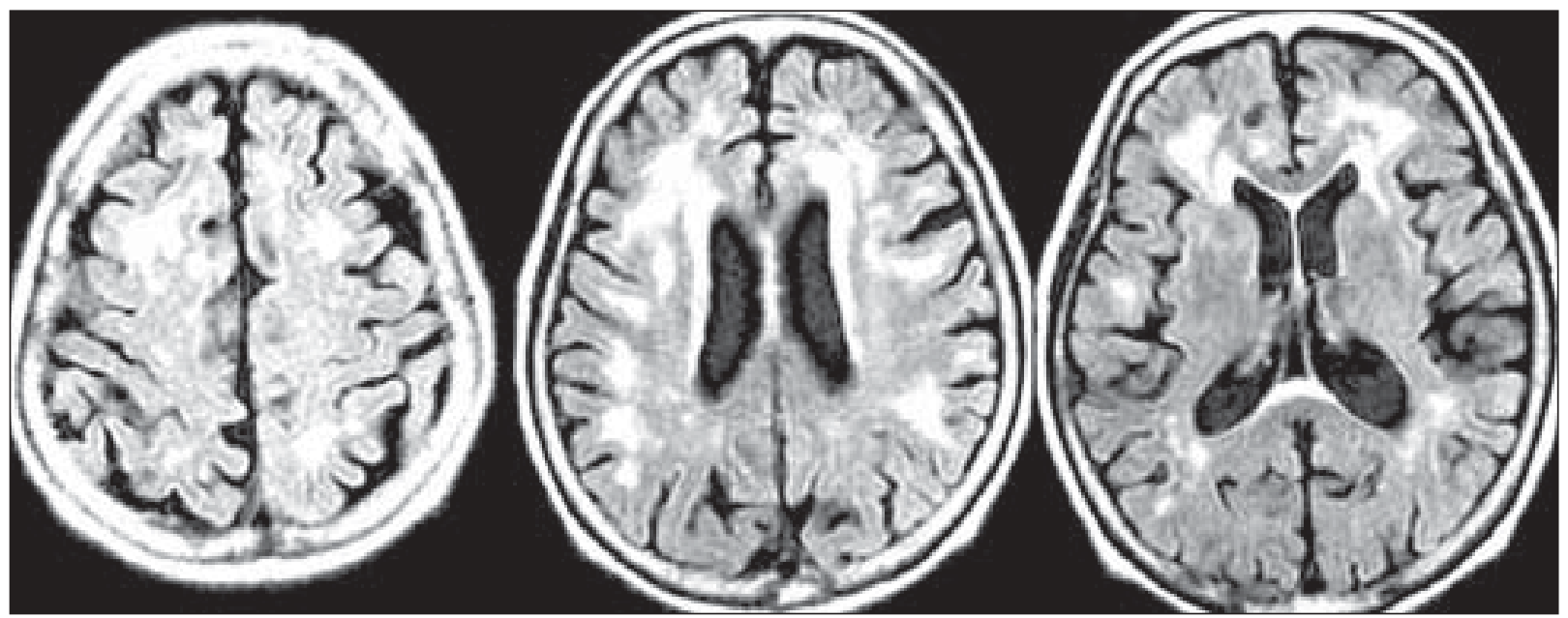

Figure 1: Multiple high intensity lesions in the white matter 


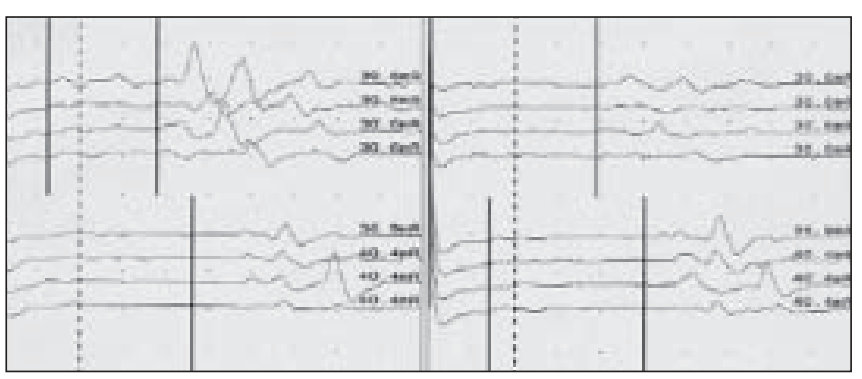

Figure 2: On right stimulation, right $\mathrm{R} 1$ and bilateral $\mathrm{R} 2$ were delayed

disease and other symptoms were due to MCTD, while all symptoms appeared in succession.

In this case, the symptoms and inflammatory response in cerebrospinal fluid and serum were ameliorated but the brain MRI after steroid therapy showed little change. This may be because the abnormal lesions in brain MRI represented irreversible damage and the symptoms in the central nervous system were ameliorated due to improvement of the encephalopathy.

Neurological problems sometimes seen in MCTD include headache, seizures, psychosis, encephalopathy, transverse myelitis, ataxia, aseptic meningitis, monocular blindness, trigeminal sensory neuropathy, polyneuropathy and entrapment neuropathy. ${ }^{[1,8,9]}$ The neurologic features observed tend to be characteristic of the dominant clinical syndrome exhibited by each patient. For example, trigeminal and peripheral neuropathy tend to occur in MCTD patients with predominant scleroderma features, while optic neuropathy, transverse myelitis, encephalopathy and ataxia occur in patients with prominent SLE features. ${ }^{[1]}$

In this case, encephalopathy and severe neuropathy might be due to systemic vasculitis as a manifestation of MCTD. Although vasculitis is a rare feature of MCTD, there were some reports about concomitant vasculitis in patients with MCTD. ${ }^{[10,11]}$ It might account for the mononeuritis multiplex pattern peripheral neuropathy and the brain MRI findings. However, because tissue biopsy was not done in this patient, the presence of vasculitis was not confirmed in this patient.
Central nervous disorders in MCTD patients are rare, and MCTD has been never discussed in the context of "treatable dementia". Many factors have been reported to contribute to "treatable dementia", including cerebrovascular disease, tumor, normal pressure hydrocephalus, toxicity, depression, encephalopathy, multiple sclerosis, sarcoidosis, hormonal abnormalities, vitamin insufficiency, organ failure, Behçet's disease, and collagen disease. ${ }^{[12]}$ In collagen disease, symptoms such as SLE, temporal arteritis, and Sjögren syndrome have been described as a rare cause of "treatable dementia" ${ }^{[12]}$ We described here "treatable dementia" due to MCTD, which while it may be rare state, it has corresponding significance because of the fact: that it is indeed treatable. Further studies will be needed to better understand the circumstances surrounding this condition.

\section{References}

1. Nadeau SE. Neurologic manifestations of connective tissue disease. Neurol Clin $2002 ; 20: 151-78$.

2. Miyata M, Nishimaki T. Neural disorders in mixed connective tissue disease (MCTD) and overlap syndrome. Nihon Rinsho Bessatsu Nihon Rinsho e! 2000;279-81.

3. Sharp GC, Irvin WS, Tan EM, Gould RG, Holman RH. Mixed connective tissue disease - an apparently distinct rheumatic disease syndrome associated with a specific antibody to an extractable nuclear antigen (ENA). Am J Med 1972;52:14559.

4. Lecky BR, Hughes RA, Murray NM. Trigeminal sensory neuropathy. A study of 22 cases. Brain 1987;110:1463-85.

5. Hagen NA, Stevens JC, Michet CJ Jr. Trigeminal sensory neuropathy associated with connective tissue diseases. Neurology 1990;40:891-6.

6. Haroon N, Nisha RS, Chandran V, Bharadwaj A. Pulmonary hypertension not a major feature of early mixed connective tissue disease: A prospective clinicoserological study. J Postgrad Med 2005;51:104-8.

7. Marvisi M, Ajolfi C, Civardi G, Delsignore R. Thyroid dysfunction and pulmonary hypertension. Recenti Prog Med 2004;95:443-6.

8. Bennett RM, O'Connell D.J. Mixed connective tisssue disease: a clinicopathologic study of 20 cases. Semin Arthritis Rheum 1980;10:25-51.

9. Bennett RM, Bong DM, Spargo BH. Neuropsychiatric problems in mixed connective tissue disease. Am J Med 1978;65:955-62.

10. Kimber TE, Scott G, Thompson PD, Beare JH. Vasculitic neuropathy and myopathy occurring as a complication of mixed connective tissue disease. Aust N Z J Med $1999 ; 29: 82-3$.

11. Graf WD, Milstein JM, Sherry DD. Stroke and mixed connective tissue disease. J Child Neurol 1993;8:256-9.

12. Abe K, Yanagihara T. Treatable dementia. Clin Neurosci 1995;13:644-5.

\section{Accepted on 09-11-2005}

